This is an electronic reprint of the original article. This reprint may differ from the original in pagination and typographic detail.

Author(s): Garaud, Julien; Silaev, Mikhail; Babaev, Egor

Title: $\quad$ Change of the vortex core structure in two-band superconductors at the impurityscattering-driven $\mathrm{s} \pm / \mathrm{s}++$ crossover

Year: $\quad 2017$

Version:

Please cite the original version:

Garaud, J., Silaev, M., \& Babaev, E. (2017). Change of the vortex core structure in two-band superconductors at the impurity-scattering-driven $\mathrm{s} \pm / \mathrm{s}++$ crossover. Physical Review B, 96(14), 140503(R). https://doi.org/10.1103/physrevb.96.140503

All material supplied via JYX is protected by copyright and other intellectual property rights, and duplication or sale of all or part of any of the repository collections is not permitted, except that material may be duplicated by you for your research use or educational purposes in electronic or print form. You must obtain permission for any other use. Electronic or print copies may not be offered, whether for sale or otherwise to anyone who is not an authorised user. 


\title{
Change of the vortex core structure in two-band superconductors at the impurity-scattering-driven $s_{ \pm} / s_{++}$crossover
}

\author{
Julien Garaud, ${ }^{1}$ Mihail Silaev, ${ }^{2}$ and Egor Babaev ${ }^{1}$ \\ ${ }^{1}$ Department of Physics, KTH-Royal Institute of Technology, Stockholm, SE-10691 Sweden \\ ${ }^{2}$ Department of Physics and Nanoscience Center, University of Jyväskylä, P.O. Box 35 (YFL), FI-40014 University of Jyväskylä, Finland
}

(Received 20 July 2017; published 6 October 2017)

\begin{abstract}
We report a nontrivial transition in the core structure of vortices in two-band superconductors as a function of interband impurity scattering. We demonstrate that, in addition to singular zeros of the order parameter, the vortices there can acquire a circular nodal line around the singular point in one of the superconducting components. It results in the formation of the peculiar "moat"-like profile in one of the superconducting gaps. The moat-core vortices occur generically in the vicinity of the impurity-induced crossover between $s_{ \pm}$and $s_{++}$states.
\end{abstract}

DOI: 10.1103/PhysRevB.96.140503

Singularities that typically occur in quantum vortices are pointlike: i.e., in two dimensions, the modulus of the complex order parameter (the density of superconducting electrons) vanishes at some point in the vortex core [1-3]. We consider qualitatively different vortex structures in a rather generic, and microscopically simple model of a two-band superconductor with impurities. In such a system, vortices can have a circular nodal line where the superconducting gap function in one of the bands vanishes. In three dimensions it extends to a cylindrical nodal surface surrounding the vortex line. We introduce the name "moat-core"-vortex to distinguish such an exotic structure, shown schematically in Figs. 1(b) and 1(c), from the usual two-component vortices with monotonic gap profiles [Figs. 1(a) and 1(d)].

Two-band superconductors where the pairing is generated by interband electron-electron repulsion [4], tend to form the so-called $s_{ \pm}$superconducting state with a sign change between the gap functions in different bands [5,6] $\Delta_{1}$ and $\Delta_{2}$. Namely, there is a $\pi$ relative phase between the components $\left|\Delta_{j}\right| e^{i \theta_{j}}$ of order parameter for the band index $j=1,2$. Thus, in contrast to the $s_{++}$state where the ground-state phase difference $\theta_{12} \equiv$ $\theta_{2}-\theta_{1}$ is zero, the $s_{ \pm}$state has $\theta_{12}=\pi$. Increasing disorder in dirty two-band superconductors rather generically leads to a crossover from the $s_{ \pm}$to the $s_{++}$state.

For the simplest two-band weak-coupling theory, the crossover can be of two types. The first is a direct one [7] involving a continuous sign change in one of the gap functions, e.g., $\Delta_{1}$. Hereafter we call $\Delta_{1}$ the subdominant component, since near the critical temperature $T_{c}$, it can be considered as induced by the stronger gap $\Delta_{2}$ due to the Cooper pair interband tunneling. The subdominant gap function amplitude vanishes at the crossover line, while the leading component $\Delta_{2}$ remains nonzero. The second scenario involves the intermediate time-reversal symmetry breaking $s+i s$ state [8], when both gap functions $\Delta_{1,2}$ are finite but acquire a nontrivial phase difference $\theta_{12} \neq \pi n$. Quantitative study has shown that in the second scenario, the intermediate $s+i s$ state occupies a vanishingly small region of the phase diagram [9] (see also note [10]). At the same time, the signature of the $s_{ \pm} / s_{++}$crossover has recently been experimentally observed in the superconducting compound from the iron-pnictide family with controlled disorder [15].

Here we consider vortex solutions near the $s_{ \pm} / s_{++}$ crossover line, and demonstrate the formation of moat-core vortices featuring a nonmonotonic order parameter distribution, and a circular (or cylindrical) nodal line where $\Delta_{1}\left(r_{0}\right)=0$. We calculate superconducting ground states and vortex structures within the weak-coupling model of two-band superconductors with a high concentration of impurities. Such system can be described by two coupled Usadel equations with interband impurity scattering terms [16]:

$$
\begin{aligned}
\omega_{n} f_{i}= & \frac{D_{i}}{2}\left(g_{i} \boldsymbol{\Pi}^{2} f_{i}-f_{i} \nabla^{2} g_{i}\right)+\Delta_{i} g_{i} \\
& +\sum_{j \neq i} \gamma_{i j}\left(g_{i} f_{j}-g_{j} f_{i}\right) .
\end{aligned}
$$

Here $\omega_{n}=(2 n+1) \pi T, n \in \mathbb{Z}$ are the fermionic Matsubara frequencies, and $T$ the temperature. $D_{i}$ are the electron diffusivities, and $\gamma_{i j}$ the interband scattering rates. Propagators in each band obey the normalization condition $\left|f_{i}\right|^{2}+g_{i}^{2}=1$, where the quasiclassical propagators $f_{i}$ and $g_{i}$ are, respectively, the anomalous and normal Green's functions. The gap
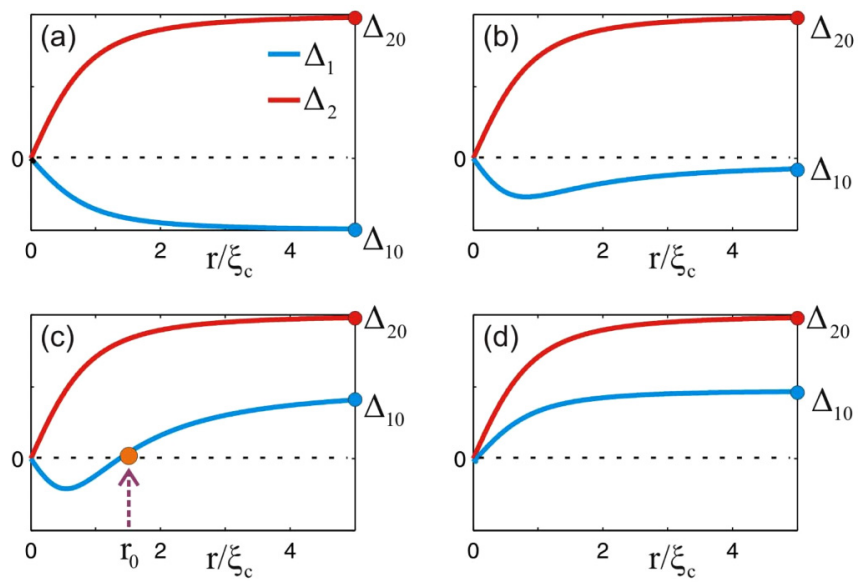

FIG. 1. Schematic picture illustrating the evolution of gap function profiles $\Delta_{1,2}(r)$ near the vortex cores, in two-band superconductors when the bulk state undergoes the $s_{ \pm} / s_{++}$crossover. Panels (a) and (d) display the usual vortex profiles in the $s_{ \pm}$and $s_{++}$ phases, respectively. Panel (b) shows a vortex with overshooting nonmonotonic behavior of the subdominant component $\Delta_{1}(r)$, while panel (c) displays the moat-core vortex in the $s_{++}$phase with the node $\Delta_{1}\left(r_{0}\right)=0$. 
functions are determined by the self-consistency equations

$$
\Delta_{i}=2 \pi T \sum_{n=0}^{N_{d}} \sum_{j} \lambda_{i j} f_{j}\left(\omega_{n}\right),
$$

for the Green's functions that satisfy Eq. (1). Here $N_{d}=$ $\Omega_{d} /(2 \pi T)$ is the summation cutoff at Debye frequency $\Omega_{d}$. The diagonal elements $\lambda_{i i}$ of the coupling matrix $\hat{\lambda}$ in the self-consistency equation (2), describe the intraband pairing. The interband interaction is determined by the off-diagonal terms $\lambda_{i j}(j \neq i)$ which can be either positive or negative.

An expansion in small $\left|\Delta_{j}\right| \ll T_{c}$ and their gradients gives the Ginzburg-Landau (GL) model:

$$
\begin{aligned}
\frac{\mathcal{F}}{\mathcal{F}_{0}}= & \sum_{j=1}^{2}\left\{\frac{k_{j j}}{2}\left|\Pi \Delta_{j}\right|^{2}+a_{j j}\left|\Delta_{j}\right|^{2}+\frac{b_{j j}}{2}\left|\Delta_{j}\right|^{4}\right\} \\
& +\frac{k_{12}}{2}\left[\left(\Pi \Delta_{1}\right)^{*} \Pi \Delta_{2}+\left(\Pi \Delta_{2}\right)^{*} \Pi \Delta_{1}\right] \\
& +2\left(a_{12}+c_{11}\left|\Delta_{1}\right|^{2}+c_{22}\left|\Delta_{2}\right|^{2}\right) \operatorname{Re}\left(\Delta_{1}^{*} \Delta_{2}\right) \\
& +\left(b_{12}+c_{12} \cos 2 \theta_{12}\right)\left|\Delta_{1}\right|^{2}\left|\Delta_{2}\right|^{2}+\frac{\boldsymbol{B}^{2}}{2} .
\end{aligned}
$$

The two gaps in the different bands are electromagnetically coupled by the vector potential $\boldsymbol{A}$ of the magnetic field $\boldsymbol{B}=\boldsymbol{\nabla} \times \boldsymbol{A}$, through the covariant derivative $\boldsymbol{\Pi} \equiv \boldsymbol{\nabla}+i q \boldsymbol{A}$ where $q$ is the electromagnetic coupling constant that parametrizes the magnetic field penetration depth. The two components are also directly coupled via potential terms in the third and fourth lines of Eq. (3), and via the mixed-gradient term, line 2. The coefficients of the Ginzburg-Landau functional $a_{i j}, b_{i j}, c_{i j}$, and $k_{i j}$ can be calculated microscopically. We list here only the expressions for the gradient terms, which are crucial for the correct determination of the transition in vortex structure:

$$
\begin{aligned}
& k_{i i}=2 \pi T N_{i} \sum_{n=0}^{N_{d}} \frac{D_{i}\left(\omega_{n}+\gamma_{j i}\right)^{2}+\gamma_{i j} \gamma_{j i} D_{j}}{\omega_{n}^{2}\left(\omega_{n}+\gamma_{i j}+\gamma_{j i}\right)^{2}}, \\
& k_{i j}=2 \pi T N_{i} \gamma_{i j} \sum_{n=0}^{N_{d}} \frac{D_{i}\left(\omega_{n}+\gamma_{j i}\right)+D_{j}\left(\omega_{n}+\gamma_{i j}\right)}{\omega_{n}^{2}\left(\omega_{n}+\gamma_{i j}+\gamma_{j i}\right)^{2}},
\end{aligned}
$$

with $j \neq i$, and $N_{i}=\lambda_{j i} /\left(\lambda_{12}+\lambda_{21}\right)$ are the partial densities of states. Note that the regimes considered below are with symmetric interband coupling, thus implying that $N_{2} / N_{1}=1$. The coefficients given by Eq. (4) satisfy the condition $k_{11} k_{22}-k_{12}^{2}>0$ yielding the positively defined gradient energy in Eq. (3) for the entire range of diffusivities $D_{1,2}$ and effective interband scattering rate $\Gamma=\gamma_{12} / N_{2}=$ $\gamma_{21} / N_{1}$. The previously reported expressions [16] for $k_{i j}$ violate this condition and therefore in general they can be used only for the infinitesimally small values of $\Gamma$. For the calculations we use dimensionless variables, normalizing the gaps by $T_{c}$, the lengths by $\xi_{0}=\sqrt{D_{1} / T_{c}}$, the magnetic field by $B_{0}=T_{c} \sqrt{4 \pi \nu_{1}}$, and the free energy $\mathcal{F}_{0}=B_{0}^{2} / 4 \pi$, where $v_{1}$ is the density of states in the first band. The electromagnetic coupling constant is $q=2 \pi B_{0} \xi_{0}^{2} / \Phi_{0}$. In these units, the London penetration length $\lambda_{L}$ is given by $\lambda_{L}^{-2}=q^{2}\left(k_{i i} \Delta_{i 0}^{2}+2 k_{12} \Delta_{10} \Delta_{20}\right)$, where $\Delta_{i 0}$ is the bulk value of the dimensionless gap.
The bulk phase diagram given by this model was calculated both at the GL level and verified against the numerical solution of the full Usadel theory in [9]. For temperatures rather close to $T_{c}$ it typically displays a direct $s_{ \pm} / s_{++}$crossover line which is rather featureless with respect to thermodynamic signatures [9]. Below, we demonstrate that there is nevertheless a transition in vortex core structure across that line. This could have a number of consequences for behavior of the system in external magnetic fields.

To investigate the properties of single vortex solutions, the physical degrees of freedom $\Delta_{1,2}$ and $\boldsymbol{A}$ are discretized within a finite-element formulation [17], and the Ginzburg-Landau free energy (3) is minimized using a nonlinear conjugate gradient algorithm. Given an initial guess where both components have the same phase winding (at large distances $\Delta_{i} \propto e^{i \theta}$ and $\theta$ is the polar angle relative to the vortex center), the minimization procedure leads, after convergence of the algorithm, to a vortex configuration [18]. Figure 2 shows the numerically calculated single vortex solutions in the vicinity of the impurity-induced crossover, in the case of a two-band superconductor with nearly degenerate bands and weak repulsive interband pairing interaction. There is a transition in the vortex profiles of the subdominant component $\Delta_{1}$ when approaching the $s_{ \pm} / s_{++}$ crossover line. First, we observe that on the $s_{ \pm}$side of the crossover, $\Delta_{1}(r)$ distribution exhibits a strong increase near the core, strongly overshooting its ground-state value $\Delta_{10}$ which is retained asymptotically at $r \rightarrow \infty$. We note that a small density overshoot effect was also obtained in the two-band model with ballistic and diffusive bands [19]. Here we point out that in the vicinity of $s_{++} / s_{ \pm}$crossover, the near-core overshoot can be very large, reaching about $100 \%$ of the subdominant ground-state amplitude $\Delta_{10}$ (see the examples in Fig. 2). The effect should be present rather generically in the presence of the interband impurity scattering since it originates from the mixed-gradient term in Eq. (5) that tends to become negative. Correspondingly, in the presence of strong superconducting currents it becomes under certain conditions energetically beneficial to increase density.

We find that near the $s_{ \pm} / s_{++}$crossover there is a rather generic effect of formation of the circular nodal lines of the subdominant component $\Delta_{1}=0$. The nodal lines exist in addition to the usual point singularities at the vortex center. In that regime, the ground state is $s_{++}$so that the interband phase difference disappears far from the vortex center $\theta_{12} \rightarrow 0$. However, due to the competition between gradient and Josephson terms, it is more favorable to achieve a $\theta_{12}=\pi$ ( $s_{ \pm}$state $)$, in the vicinity of the core singularity. The transition between the localized "core" states with $\theta_{12}=\pi$ and the asymptotic states $\theta_{12}=0$ is realized by nullifying the subdominant gap $\Delta_{1}\left(r_{0}\right)=0$ at a given distance $r_{0}$ from the center, when the Josephson energy term wins over the gradient one. The effect should also be generic for a wide range of models with this structure of the gradient terms competing with the intercomponent Josephson coupling [20]. We discuss below that the effect should be stronger at lower temperatures and is underestimated by the GL model.

The tendency for the formation of a localized $s_{ \pm}$state inside the vortex core can be qualitatively understood by analyzing the functional (3). The structure of axially symmetric vortices is given by the ansatz for the order parameter components 

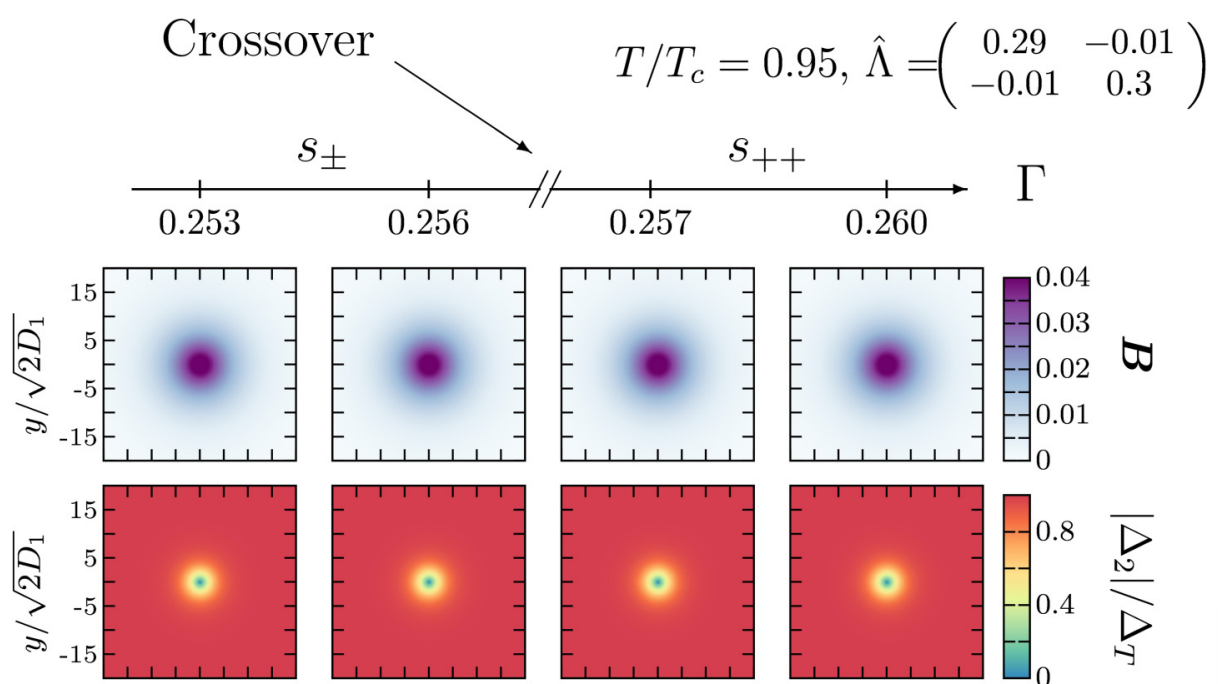

\section{$\varpi$}
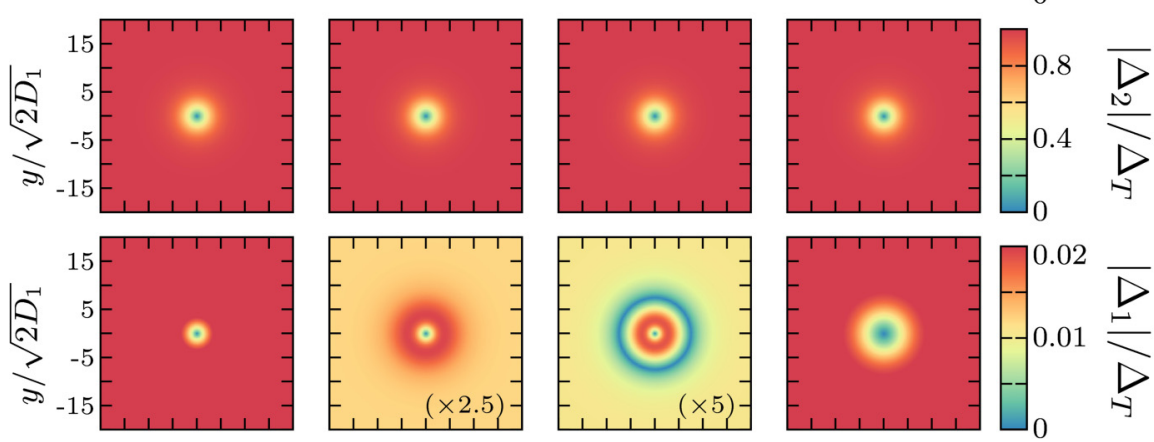

\begin{tabular}{|c||c|c|c|c|}
\hline$\Gamma$ & 0.253 & 0.256 & 0.257 & 0.260 \\
\hline \hline$a_{11}\left(\times 10^{-2}\right)$ & 3.463 & 3.462 & 3.461 & 3.460 \\
\hline$a_{22}\left(\times 10^{-2}\right)$ & -2.291 & -2.292 & -2.292 & -2.294 \\
\hline$a_{12}\left(\times 10^{-3}\right)$ & -0.161 & -0.869 & -1.105 & -1.813 \\
\hline $\begin{array}{c}b_{11}= \\
b_{22}\left(\times 10^{-2}\right)\end{array}$ & 7.413 & 7.422 & 7.425 & 7.435 \\
\hline$b_{12}\left(\times 10^{-3}\right)$ & -2.804 & -2.835 & -2.845 & -2.876 \\
\hline \hline $\left.\begin{array}{c}c_{11}= \\
c_{22}\left(\times 10^{-3}\right)\end{array}\right)$ & 3.290 & 3.334 & 3.348 & 3.392 \\
\hline$c_{12}\left(\times 10^{-4}\right)$ & 3.137 & 3.219 & 3.246 & 3.330 \\
\hline \hline $\begin{array}{c}k_{11}= \\
k_{22}\left(\times 10^{-1}\right)\end{array}$ & 4.578 & 4.581 & 4.582 & 4.584 \\
\hline$k_{12}\left(\times 10^{-2}\right)$ & 3.735 & 3.784 & 3.800 & 3.849 \\
\hline \hline$\lambda_{L}\left(\times 10^{1}\right)$ & 1.0602 & 1.0630 & 1.0636 & 1.0631 \\
\hline \hline
\end{tabular}


FIG. 2. Transition in vortex solutions in the vicinity of the impurity-induced crossover line of a two-band superconductor with nearly degenerate bands and weak repulsive interband pairing interaction $\left(\lambda_{11}=0.29, \lambda_{22}=0.3\right.$, and $\left.\lambda_{12}=\lambda_{21}=-0.01\right)$, and with equal electron diffusivities $\left(D_{2} / D_{1}=1\right)$. The temperature is $T / T_{c}=0.95, q=0.25$, and tuning the strength of the effective interband impurity scattering drives the system from bulk $s_{ \pm}$to bulk $s_{ \pm} s_{++}$. The table indicates the calculated values of the London penetration depth $\Lambda_{L}$ and of the coefficients of the Ginzburg-Landau free energy. The different lines respectively display the magnetic field $\boldsymbol{B}$, the larger gap $\left(\Delta_{2}\right)$, and the smaller gap $\left(\Delta_{1}\right)$. The last line shows the relative phase $\theta_{12}$ that specifies whether the superconducting ground state is $s_{++}$or $s_{ \pm}$. The third column shows a vortex solution that has a pointlike and a "moat"-like zero in $\Delta_{1}$. Note this is a close-up view of the vortex core which is actually calculated in larger grids.

$\Delta_{j}(\boldsymbol{r})=\tilde{\Delta}_{j}(r) e^{i \theta}$, where $\tilde{\Delta}_{j}(r)$ are the real-valued profiles of the order parameter components and the polar coordinates $r, \theta$ are determined relative to the vortex center. In this case the GL energy contribution from the mixed-gradient term can be written as follows:

$$
\begin{aligned}
F_{G} & \equiv \frac{k_{12}}{2}\left[\left(\Pi \Delta_{1}\right)^{*} \Pi \Delta_{2}+\text { c.c. }\right] \\
& =k_{12}\left(\nabla_{r} \tilde{\Delta}_{1} \nabla_{r} \tilde{\Delta}_{2}+r^{-2} \tilde{\Delta}_{1} \tilde{\Delta}_{2}\right)
\end{aligned}
$$

where the vector potential contribution is neglected since it is small inside the vortex core. This term describes the interaction between the order parameter components which is qualitatively similar to the interband Josephson energy contribution in Eq. (3):

$$
F_{J} \equiv 2\left(a_{12}+c_{11}\left|\tilde{\Delta}_{1}\right|^{2}+c_{22}\left|\tilde{\Delta}_{2}\right|^{2}\right) \tilde{\Delta}_{1} \tilde{\Delta}_{2} .
$$

In the bulk phase, where the gradient energy is zero, $F_{G}=0$, the phase locking corresponds to the $s_{++} / s_{ \pm}$state depending on the sign of the effective Josephson coupling
$J=a_{12}+c_{11}\left|\tilde{\Delta}_{1}\right|^{2}+c_{22}\left|\tilde{\Delta}_{2}\right|^{2}$. The crossover line can be defined parametrically in the $\Gamma, T$ plane as $J(\Gamma, T)=0$. In spatially nonhomogeneous states, e.g., in the presence of vortices, the relative sign of the gap functions $\tilde{\Delta}_{1,2}$ is determined by the local interplay of two phase-locking energies $F_{G}$ and $F_{J}$.

In the vortex cores, the order parameter profiles can be approximated by linear dependencies $\tilde{\Delta}_{j}(r) \approx r d \tilde{\Delta}_{j} / d r$, thus yielding $F_{G} \approx k_{12}\left(d \tilde{\Delta}_{1} / d r\right)\left(d \tilde{\Delta}_{2} / d r\right)$. There, since the mixed-gradient coefficient is always positive $k_{12}>0$, the energy $F_{G}$ favors the opposite signs of the order parameter slopes, e.g., $d \tilde{\Delta}_{2} / d r>0$ and $d \tilde{\Delta}_{1} / d r<0$. That leads to the opposite signs of gap functions near the vortex center $\tilde{\Delta}_{2}>0$ and $\tilde{\Delta}_{1}<0$. This tendency competes with that favored by the Josephson energy if $J<0$, corresponding to the bulk $s_{++}$phase when the gaps have the same signs far from the core. Therefore, provided that the gradient energy dominates close to the vortex center $\left(\left|F_{G}\right|>\left|F_{J}\right|\right)$, one can expect the nonmonotonic distribution for the component $\Delta_{1}(r)$, crossing zero at some finite distance $r=r_{0}$ determined by the competition of $F_{G}$ and $F_{J}$. In the two-dimensional plane 
perpendicular to the vortex line, such zero points of $\Delta_{1}\left(r_{0}\right)=0$ form the circular nodal line around the singular point at the vortex center $r=0$.

The scenario discussed above is actually generic for any two-band $s_{++}$superconductor with interband impurity scattering. It can be shown that the effect should be stronger away from the superconducting phase transition. For a system that breaks only a single symmetry, at the mean-field level only one (critical) mode survives in the limit $\tau \equiv\left(1-T / T_{c}\right) \rightarrow+0$. In general, the critical mode corresponds to a certain linear combination of the gap function fields $\Delta_{1,2}$. Even if there are other well-defined subdominant modes that are characterized by other coherence lengths, they have vanishing amplitude when $\tau$ is much smaller than other parameters in the problem [21]. In the limit $\tau \rightarrow 0$, the energy contributions can be estimated by retaining only the contribution from the dominant mode, that is, $\left|d \tilde{\Delta}_{i} / d r\right| \propto\left|\Delta_{i 0}\right| / \xi_{c}(T)$, where $\xi_{c}(T) \propto 1 / \sqrt{\tau}$ is the critical coherence length. Hence the mixed-gradient energy $\left|F_{G}\right| \propto k_{12}\left|\Delta_{10} \Delta_{20}\right| / \xi_{c}^{2}(T)$ should be compared to the Josephson energy $F_{J} \propto J \Delta_{10} \Delta_{20}$. One can see that the condition of the vortex transition $\left|F_{G}\right|>F_{J}$ is satisfied only provided that the coupling is small enough, $|J| \ll k_{12} / \xi_{c}^{2}(T)$, which certainly does not hold near the critical temperature in the limit $\tau \rightarrow 0$ when $\xi_{c}(T) \rightarrow \infty$. However, one can expect that inside the vortex core the gradient energy always dominates in the vicinity of the impurity-driven $s_{ \pm} / s_{++}$crossover where the effective Josephson coupling disappears, $J(\Gamma, T)=$ 0 . This argument heuristically explains the numerically found moat-core vortex structures shown in Fig. 2.

The existence of exotic moat-core vortices does not depend on specific values of the pairing coefficients. Indeed, we found such solutions for all the different $\hat{\Lambda}$ we investigated. Based on the above qualitative argument, one can conclude that these vortex structures inevitably appear sufficiently close to the crossover line. Moreover, we find that typically, the region of moat-core vortices in the $\Gamma, T$ phase diagram tends to become larger with the increased ratio of diffusion coefficients $D_{2} / D_{1}$. This effect can be explained by the softening of the order parameter in the subdominant band which facilitates the formation of additional zeros in the $\Delta_{1}(r)$ gap distribution.

In conclusion, we have shown that there is a vortex structure transition across the $s_{ \pm} / s_{++}$crossover line driven by the impurity scattering, in two-band superconductors. On the $s_{ \pm}$ side of this crossover, vortices have a strong overshooting in the distribution of the subdominant component of the order parameter. On the other side, there are moat-core vortices with an $s_{ \pm}$phase inclusion in the cores, separated from the bulk $s_{++}$phase by circular nodal lines. This raises a number of interesting questions. First, it should be interesting to investigate the electronic structure of the moat-core vortices. Second, this system for the parameters close to the $s_{ \pm} / s_{++}$ crossover should have a nontrivial behavior in the external magnetic field. Indeed, in contrast to the zero-field picture of a sharp crossover, the lattice and liquids of moat-core vortices represent a macroscopic phase separation or mircoemulsionlike $s_{ \pm}$inclusions inside the $s_{++}$state. As the vortex density rises in increasing field, there should also be a field-induced crossover from $s_{++}$to the $s_{ \pm}$. This can be resolved in local phase-sensitive probes [22].

We thank J. Carlström for many discussions. The work was supported by the Swedish Research Council Grants No. 642-2013-7837 and No. VR2016-06122 and Goran Gustafsson Foundation for Research in Natural Sciences and Medicine. M.S. was supported by the Academy of Finland. The computations were performed on resources provided by the Swedish National Infrastructure for Computing (SNIC) at National Supercomputer Center at Linköping, Sweden.
[1] L. Onsager, Statistical hydrodynamics, Il Nuovo Cimento Ser. 9 6, 279 (1949).

[2] A. A. Abrikosov, On the Magnetic properties of superconductors of the second group, Zh. Eksp. Teor. Fiz. 32, 1442 (1957) [Sov. Phys. JETP 5, 1174 (1957)].

[3] R. P. Feynman, Application of quantum mechanics to liquid helium, Prog. Low Temp. Phys. 1, 17 (1955).

[4] P. J. Hirschfeld, M. M. Korshunov, and I. I. Mazin, Gap symmetry and structure of Fe-based superconductors, Rep. Prog. Phys. 74, 124508 (2011).

[5] I. I. Mazin, D. J. Singh, M. D. Johannes, and M. H. Du, Unconventional Superconductivity with a Sign Reversal in the Order Parameter of $\mathrm{LaFeAsO}_{1-x} \mathrm{~F}_{x}$, Phys. Rev. Lett. 101, 057003 (2008).

[6] A. V. Chubukov, D. V. Efremov, and I. Eremin, Magnetism, superconductivity, and pairing symmetry in iron-based superconductors, Phys. Rev. B 78, 134512 (2008).

[7] D. V. Efremov, M. M. Korshunov, O. V. Dolgov, A. A. Golubov, and P. J. Hirschfeld, Disorder-induced transition between $s_{ \pm}$ and $s_{++}$states in two-band superconductors, Phys. Rev. B 84, 180512 (2011).
[8] V. Stanev and A. E. Koshelev, Complex state induced by impurities in multiband superconductors, Phys. Rev. B 89, 100505 (2014).

[9] M. Silaev, J. Garaud, and E. Babaev, Phase diagram of dirty two-band superconductors and observability of impurity-induced $s+i s$ state, Phys. Rev. B 95, 024517 (2017).

[10] It should be noted that this statement applies only to the dirty two-band case; in the three-band case, the $s+i s$ state can occupy a much larger fraction of the phase diagram $[11,12,13]$. Its experimental observation was recently reported in [14].

[11] V. Stanev and Z. Tešanović, Three-band superconductivity and the order parameter that breaks time-reversal symmetry, Phys. Rev. B 81, 134522 (2010).

[12] S. Maiti and A. V. Chubukov, $s+i s$ state with broken timereversal symmetry in Fe-based superconductors, Phys. Rev. B 87, 144511 (2013).

[13] J. Böker, P. A. Volkov, K. B. Efetov, and I. Eremin, $s+i s$ superconductivity with incipient bands: Doping dependence and STM signatures, Phys. Rev. B 96, 014517 (2017). 
[14] V. Grinenko, P. Materne, R. Sarkar, H. Luetkens, K. Kihou, C. H. Lee, S. Akhmadaliev, D. V. Efremov, S.-L. Drechsler, and H.-H. Klauss, Superconductivity with broken time-reversal symmetry in ion-irradiated $\mathrm{Ba}_{0.27} \mathrm{~K}_{0.73} \mathrm{Fe}_{2} \mathrm{As}_{2}$ single crystals, Phys. Rev. B 95, 214511 (2017).

[15] M. B. Schilling, A. Baumgartner, B. Gorshunov, E. S. Zhukova, V. A. Dravin, K. V. Mitsen, D. V. Efremov, O. V. Dolgov, K. Iida, M. Dressel, and S. Zapf, Tracing the $s_{ \pm}$symmetry in iron pnictides by controlled disorder, Phys. Rev. B 93, 174515 (2016).

[16] A. Gurevich, Enhancement of the upper critical field by nonmagnetic impurities in dirty two-gap superconductors, Phys. Rev. B 67, 184515 (2003).

[17] F. Hecht, New development in Freefem++, J. Numer. Math. 20, 251 (2012).

[18] Being in zero external field, the vortex is created only by the initial phase winding configuration. For further details on the numerical methods employed here; see, for example, a related discussion in J. Garaud, E. Babaev, T. A. Bojesen, and A. Sudbø, Lattices of double-quanta vortices and chirality inversion in $p_{x}+i p_{y}$ superconductors, Phys. Rev. B 94, 104509 (2016).
[19] K. Tanaka, M. Eschrig, and D. F. Agterberg, Theory of vortices in hybridized ballistic/diffusive-band superconductors, Phys. Rev. B 75, 214512 (2007).

[20] Here we focus on the role of impurity-induced gradient terms. In fact, the effect should be more general: compare with the phenomenological discussion of vortex structure in three-component models without mixedgradient terms but with frustrated interband coupling in J. Carlström, J. Garaud, and E. Babaev, Length scales, collective modes, and type-1.5 regimes in three-band superconductors, Phys. Rev. B 84, 134518 (2011).

[21] See a detailed discussion of the behavior of dominant and subdominant modes in multiband superconductors in the limit $\tau \equiv(1-T / T c) \rightarrow+0$ in $\mathrm{M}$. Silaev and E. Babaev, Microscopic derivation of two-component Ginzburg-Landau model and conditions of its applicability in two-band systems, Phys. Rev. B 85, 134514 (2012).

[22] P. J. Hirschfeld, D. Altenfeld, I. Eremin, and I. I. Mazin, Robust determination of the superconducting gap sign structure via quasiparticle interference, Phys. Rev. B 92, 184513 (2015). 